\title{
Quasi-reflexive Fréchet spaces and contractively power bounded operators
}

\author{
Krzysztof PISZCZEK
}

\begin{abstract}
In this paper we introduce a new class of operators acting on a locally convex space. We show that for some Fréchet spaces all these operators are mean ergodic. This leads to the conclusion that the classes of reflexive and non-reflexive Fréchet spaces are, in a sense, close to each other.
\end{abstract}

Mathematics Subject Classification (2010). Primary 46A04, 47A35.

Keywords. Fréchet space, Quasi-reflexive space, Mean ergodic operator, Power bounded operator.

1. Introduction. In [3] the following definition is introduced: a Banach space $X$ is called quasi-reflexive (of order $n$ ) if $\operatorname{codim}_{X^{\prime \prime}} \pi(X)<+\infty\left(\operatorname{codim}_{X^{\prime \prime}} \pi(X)=\right.$ $n$ ), shortly $n$-quasi-reflexive. This definition arose from the very well known paper of R. C. James who constructed in [9] an example of a non-reflexive Banach space $X$ of codimension 1 in its bidual. Properties of such spaces have been studied in $[4,8,10,17,18]$. One of the outstanding profits of these spaces is that they have served as examples to settle several conjectures. Recently Fonf, Lin, and Wojtaszczyk have constructed in [7] a quasi-reflexive Banach space of order 1 with all contractions on the space itself and on its dual mean ergodic. Thus answering negatively a question of Sucheston posed in [19].

The aim of this paper is to consider (after introducing a reasonable notation) an analogous problem in the Fréchet space setting. Here the notion of quasi-reflexivity is also available since, whenever we start with an arbitrary Fréchet space $X$, by $\left[15,25.3\right.$ and 25.10] all the spaces $\pi(X), X^{\prime \prime}$ and $X^{\prime \prime} / \pi(X)$ are Fréchet. Examples of such spaces without infinite-dimensional Banach subspaces may be found in [14]. Let us recall them for the convenience of the

The research of the author has been supported in the years 2007-2010 by the Ministry of Science and Higher Education, Poland, Grant no. N N201 274033. 
reader. If $J_{p}$ denotes the 1 -quasi-reflexive James space with the $\ell_{p}$-norm then $J_{p+}:=\operatorname{proj}_{q>p} J_{q}$ for $1 \leqslant p<+\infty$ is a 1-quasi-reflexive Fréchet space while $J_{p-}:=\operatorname{ind}_{1<q<p} J_{q}$ for $1<p \leqslant+\infty$ is a 1 -quasi-reflexive LB-space. In what follows we will adapt as much of the Banach viewpoint as possible but in several situations the knowledge on the structure of locally convex spaces will be of particular importance.

The paper is divided into five parts. In Section 2 we recall the notion of mean ergodicity together with a short description of most important results. Next we collect several observations on operators on quasi-reflexive spaces of order 1. Section 3 is devoted to obtaining a specific fundamental sequence of bounded sets and (independently) a specific basis of zero neighbourhoods in $X$ or $X^{\prime}$ whenever $X$ is a separable real Fréchet space which is quasi-reflexive of order 1. In Section 4 we define contractively power bounded operators and show when such operators are mean ergodic. The last Section contains the main result of the paper which gives examples of non-reflexive Fréchet spaces so that all contractively power bounded operators are mean ergodic. Combining these results with [6] we see that the classes of reflexive and non-reflexive Fréchet spaces are, in a sense, close to each other.

The general reference for functional analysis is [15] and for ergodic theory we refer the reader to [13].

2. Preliminaries. Recall that a continuous and linear operator $T$ on a Banach space $X$ is called mean ergodic (see, e.g., [13, §2.1],) if the limits

$$
P x:=\lim _{n \rightarrow+\infty} \frac{1}{n} \sum_{k=1}^{n} T^{k} x
$$

exist for every element $x \in X$. It is called power bounded if

$$
\sup _{k \in \mathbb{N}}\left\|T^{k}\right\|<+\infty .
$$

If every power bounded operator is mean ergodic then the space $X$ is called mean ergodic. In 1937 von Neumann proved that unitary operators on Hilbert spaces are mean ergodic and a year later F. Riesz showed that all $L^{p}$-spaces, $1<p<+\infty$, are mean ergodic. In 1939 Lorch proved that all reflexive Banach spaces are mean ergodic and raised the question of whether the converse holds. The affirmative answer for spaces with bases is given in [6]. For more informations on that topic see [5, Chapter VIII, Section 4] and [13, Chapter 2].

Observe that (1) makes sense in any locally convex Hausdorff space (abbreviated $l c H s$ ) therefore we call an operator $T$ on such a space mean ergodic if it satisfies (1). Condition (2) needs a reformulation and we say that an operator $T$ on a lcHs is power bounded if the sequence $\left(T^{k}\right)_{k \in \mathbb{N}} \subset L(X)$ is equicontinuous. Recall that a lcHs $X$ is called quasi-reflexive if it is quasi-barrelled and $\operatorname{codim}_{X^{\prime \prime}} \pi(X)<+\infty$.

Let us now focus on Fréchet spaces that is metrizable and complete lcHs'. The topology of such a space can always be given by a non-decreasing sequence of seminorms and one can choose a countable basis of zero neighbourhoods 
consisting of closed unit balls in each of these seminorms. Thus we can always represent a Fréchet space $X$ as a projective limit of a sequence of Banach spaces $\left(X_{n}\right)_{n \in \mathbb{N}}$, i.e. $X=\operatorname{proj}_{n} X_{n}$. By $\iota_{n}: X \rightarrow X_{n}$ we denote the canonical projection and we assume without loss of generality that $\overline{\iota_{n}(X)}=X_{n}$. Suppose now that $X$ is a separable and 1-quasi-reflexive Fréchet space which can be represented as the reduced projective limit of a sequence $\left(X_{n}\right)_{n}$ of separable and 1-quasi-reflexive Banach spaces. We point out that the space $J_{p+}$ satisfies all these requirements. But a quasi-reflexive lcHs $X$ is not necessarily a closed subspace of a countable product of quasi-reflexive Banach spaces (see [20, Theorem 6.2]). Let us also remark that, if $X$ is given the above representation as a reduced projective limit, its strong dual $X_{\beta}^{\prime}$ is not necessarily the reduced projective limit of some system of 1-quasi-reflexive Banach spaces. But since $X^{\prime \prime}$ is separable, also $X^{\prime}$ is separable and hence, by $[11, \S 29.3(12)$ a)] and $[11, \S 29.4(3)] X^{\prime}=\operatorname{ind}_{n} X_{n}^{\prime}$ with $\iota_{n}^{\prime}: X_{n}^{\prime} \hookrightarrow X^{\prime}$ being linking maps. So, $X^{\prime}=$ $\operatorname{ind}_{n} X_{n}^{\prime}$ is a complete LB-space and hence, by [2, p. 57] $X^{\prime \prime}=\operatorname{proj}_{n} X_{n}^{\prime \prime}$. Moreover, for every $n \in \mathbb{N}$ we have $\overline{\iota_{n}^{\prime \prime}\left(X^{\prime \prime}\right)}=X_{n}^{\prime \prime}$ because $X$ and $X_{n}$ are quasireflexive of order 1 and $\overline{\iota_{n}(X)}=X_{n}$. In such a case all the spaces $X, X_{n}$ together with their duals and biduals are separable. Let us point out that whenever $X$ will be a separable and quasi-reflexive Fréchet space of order one, it will always be assumed that such a space can be represented as the reduced projective limit of a sequence of separable and 1-quasi-reflexive Banach spaces.

The following two results are proved for Banach spaces in [7] and the proofs carry over to our setting but for the convenience of the reader we include them below.

Proposition 2.1. Let $X$ be a quasi-reflexive of order 1 Fréchet space or its dual. Then there exists a linear and continuous, multiplicative functional $q: L(X) \rightarrow$ $\mathbb{K}$ such that its kernel is exactly the set of reflexive operators on $X$.

Proof. Suppose $X$ is a Fréchet space and for arbitrary operator $T$ acting on $X$ consider its biadjoint $T^{\prime \prime}$. Since $T^{\prime \prime}(X) \subset X$, it induces an operator $\widehat{T^{\prime \prime}} \in$ $L\left(X^{\prime \prime} / X\right)$. By assumption $X^{\prime \prime} / X$ is one-dimensional therefore $\widehat{T^{\prime \prime}}$ is a multiplication by a number, say $q(T)$. The map $q$ is the desired functional and its kernel is exactly the set of those operators $T \in L(X)$ for which $T^{\prime \prime}\left(X^{\prime \prime}\right) \subset X$. And these are by definition reflexive operators. The above argument works also in the dual case.

Remark 2.2. Let $X$ be a quasi-reflexive of order 1 Fréchet space. Then, every $T \in L(X)$ has a unique decomposition $T=\lambda I+R$, where $\lambda \in \mathbb{K}$ and $R$ is a reflexive operator.

Proposition 2.3. Let $X$ be a quasi-reflexive of order 1 Fréchet space. Suppose $T=\lambda I+R: X \rightarrow X$ is power bounded and $\lambda \neq 1$. Then both $T$ and $T^{\prime}$ are mean ergodic.

Proof. Denote by $F(T)$ the set of fixed points of $T$ and observe that $F(T)=$ $\{x \in X: R x=(1-\lambda) x\}, \quad F\left(T^{\prime \prime}\right)=\left\{x^{\prime \prime} \in X^{\prime \prime}: R^{\prime \prime} x^{\prime \prime}=(1-\lambda) x^{\prime \prime}\right\}$. Since $R^{\prime \prime}\left(X^{\prime \prime}\right) \subset X$ we have $F(T)=F\left(T^{\prime \prime}\right)$. Using the Hahn-Banach Theorem we 
see that $F\left(T^{\prime}\right)$ always separates $F(T)$. Therefore in our case $F(T)$ separates $F\left(T^{\prime}\right)$ and by Sine's Criterion [16, Theorem 13] (compare [13, Theorem 1.4]) $T$ is mean ergodic. On the other hand $F\left(T^{\prime}\right)$ separates $F\left(T^{\prime \prime}\right)$ and again by Sine's Criterion $T^{\prime}$ is mean ergodic.

3. Fréchet spaces and their duals with specifically distinguished sets. From now on all the spaces are assumed to be vector spaces over $\mathbb{R}$.

We start by recalling the following result which will be crucial for our constructions.

Proposition 3.1. ([7]) Let $X$ be a non-reflexive Banach space with separable bidual. Then there exist an equivalent norm $\| \cdot \cdot|| \mid$ on $X$, a norm 1 functional $f_{0} \in X^{\prime \prime} \backslash X$ and a norm 1 functional $F_{0} \in X^{\prime \prime \prime} \cap X^{\perp}$ such that

(i) $F_{0}\left(f_{0}\right)=1$ and $F(g)<1$ for any $g \in B_{\left(X^{\prime \prime},|| \cdot||||\right)}, g \neq f_{0}$,

(ii) if $\|H\| \|_{X^{\prime \prime \prime}}=1$ and $H\left(f_{0}\right)=1$ then $H \in X^{\perp}$.

Moreover, if $X=Y^{\prime}$ (isometrically) then $\|\cdot\| \cdot \|$ is a dual norm to a certain norm on $Y$.

Remark 3.2. (1) If $X$ is quasi-reflexive of order 1 then $F_{0}$ is the only norm 1 functional with $F_{0}\left(f_{0}\right)=1$ since, by assumption, $X^{\prime \prime}=X \oplus\left[f_{0}\right]$.

(2) By the proof of this result (see [7, Proposition 3] for details) we may take $f_{0}$ from a previously fixed dense subset.

Suppose now $X$ is a separable and quasi-reflexive of order 1 Fréchet space which can be represented as the reduced projective limit of separable and 1-quasi-reflexive Banach spaces. According to the discussion at the beginning of the previous Section $X=\operatorname{proj}_{U \in \mathcal{U}} X_{U}$ where $\mathcal{U}$ is a countable basis of zero neighbourhoods in $X$ and $X^{\prime \prime}=\operatorname{proj}_{U \in \mathcal{U}} X_{U}^{\prime \prime}$. Recall that for every zero neighbourhood $U$ in $X$ the bipolar $U^{\circ \circ}$ (each of which is taken in the consecutive dual) is a zero neighbourhood in $X^{\prime \prime}$ and by the Separation Theorem [11, Chapter $4, \S 20,7(1)] U^{\circ \circ}=\bar{U}^{\sigma\left(X^{\prime \prime}, X^{\prime}\right)}=: U_{*}$. With the above notation in mind we are able to obtain what follows.

Proposition 3.3. Suppose $X$ is a separable and 1-quasi-reflexive real Fréchet space which can be represented as the reduced projective limit of a sequence of separable and 1-quasi-reflexive real Banach spaces. Assume moreover that $X$ possesses a continuous norm. Then there exist a countable zero neighbourhood basis $\mathcal{U}$ in $X,\left\{f_{U}\right\}_{U \in \mathcal{U}} \subset X^{\prime \prime} \backslash X,\left\{F_{U}\right\}_{U \in \mathcal{U}} \subset U_{*}^{\circ} \cap X^{\perp}$ such that for every $U \in \mathcal{U}$ the following holds:

(i) $p_{U_{*}}\left(f_{U}\right)=1$,

(ii) $F_{U}\left(f_{U}\right)=1$ and $F_{U}(g)<1$ for any $g \in U_{*}, g \neq f_{U}$,

(iii) $\quad F_{U}$ is the only functional in $U_{*}^{\circ}$ with $F_{U}\left(f_{U}\right)=1$.

Proof. By assumption $X=\operatorname{proj}_{n} X_{n}$ with all the $X_{n}$ 's being separable and 1-quasi-reflexive real Banach spaces. Since $X$ has a continuous norm we may assume that the topology of $X$ is given by a non-decreasing sequence of norms. Moreover by quasi-reflexivity of $X$ the same applies to $X^{\prime \prime}$. By Proposition 3.1 there exist for every $n \in \mathbb{N}$ : a closed absolutely convex set $V_{n}$-whose 
Minkowski functional $p_{V_{n}}$ gives the topology of $X_{n}$-together with a norm one functional $g_{n} \in X_{n}^{\prime \prime} \backslash X_{n}, G_{n} \in X_{n}^{\prime \prime \prime} \cap X_{n}^{\perp}$ with the properties listed in Proposition 3.1. If $\iota_{n}: X \rightarrow X_{n}$ denotes the canonical projection then the sets $U_{n}:=\iota_{n}^{-1} V_{n}$ determine the topology of $X$. Moreover

$$
\left(U_{n}\right)_{*}=\left(\left(\iota_{n}^{-1} V_{n}\right)^{\circ}\right)^{\circ}=\left(\overline{\iota_{n}^{\prime}\left(V_{n}^{\circ}\right)}\right)^{\circ}=\left(\iota_{n}^{\prime}\left(V_{n}^{\circ}\right)\right)^{\circ}=\left(\iota_{n}^{\prime \prime}\right)^{-1}\left(V_{n}^{\circ \circ}\right)=\left(\iota_{n}^{\prime \prime}\right)^{-1}\left(V_{n}\right)_{*} .
$$

By Remark $3.2(2)$ we may assume that $g_{n}=\iota_{n}^{\prime \prime}\left(f_{n}\right)$ with $f_{n} \in\left(U_{n}\right)_{*}$. We define $F_{n}:=G_{n} \circ \iota_{n}^{\prime \prime}$ and claim that the functionals $f_{n}, F_{n}$ meet the desired properties. Indeed,

$$
p_{\left(U_{n}\right) *}\left(f_{n}\right)=p_{\left(V_{n}\right)_{*}}\left(g_{n}\right)=1
$$

which gives the first condition. Next,

$$
F_{n}\left(f_{n}\right)=G_{n}\left(g_{n}\right)=1
$$

and if $f_{n} \neq g \in\left(U_{n}\right)_{*}$ then

$$
F_{n}(g)=G_{n}\left(\iota_{n}^{\prime \prime} g\right)<1 .
$$

The last inequality follows by the fact that $X^{\prime \prime}$ has a continuous norm and $\iota_{n}^{\prime \prime} g=\iota_{n}^{\prime \prime} f_{n}$ implies $g=f_{n}$. Therefore we obtain the second condition. To get the last one suppose $F\left(f_{n}\right)=1$ for some $F \in\left(U_{n}\right)_{*}^{\circ}$. It means that $F=G \circ \iota_{n}^{\prime \prime}$ for some $G \in\left(V_{n}\right)_{*}$ and

$$
F\left(f_{n}\right)=G\left(g_{n}\right)=1 .
$$

This implies that $G=G_{n}$ and eventually $F=F_{n}$. The proof is thereby complete.

Proposition 3.4. Suppose $X$ is a separable and 1-quasi-reflexive real Fréchet space which can be represented as the reduced projective limit of a sequence of separable and 1-quasi-reflexive real Banach spaces. Then there exist a countable fundamental system $\mathcal{B}$ of bounded sets in $X^{\prime}$ together with functionals $\left\{f_{B}\right\}_{B \in \mathcal{B}} \subset X^{\prime \prime \prime} \backslash X^{\prime},\left\{F_{B}\right\}_{B \in \mathcal{B}} \subset B_{*}^{\circ} \cap\left(X^{\prime}\right)^{\perp}$ such that for every $B \in \mathcal{B}$ the following holds:

(i) $p_{B_{*}}\left(f_{B}\right)=1$,

(ii) $F_{B}\left(f_{B}\right)=1$ and $F_{B}(g)<1$ for any $g \in B_{*}, g \neq f_{B}$,

(iii) $F_{B}$ is the only functional in $B_{*}^{\circ}$ with $F_{B}\left(f_{B}\right)=1$.

Moreover, we may additionally assume that the members of $\mathcal{B}$ are polars of zero neighbourhoods in $X$.

Proof. By Proposition 3.1 we get for every $n \in \mathbb{N}$ : a closed absolutely convex set $C_{n}=V_{n}^{\circ}$-whose Minkowski functional $p_{C_{n}}$ gives the topology of $X_{n}^{\prime}$ together with a norm one functional $g_{n} \in X_{n}^{\prime \prime \prime} \backslash X_{n}^{\prime}, G_{n} \in X_{n}^{\prime \prime \prime \prime} \cap\left(X_{n}^{\prime}\right)^{\perp}$ with the properties listed in Proposition 3.1. If $\iota_{n}: X \rightarrow X_{n}$ denotes the canonical projection then the sets $B_{n}:={\overline{\iota_{n}^{\prime}\left(C_{n}\right)}}^{\sigma\left(X^{\prime}, X\right)}$ determine a fundamental sequence of bounded sets in $X^{\prime}$. Moreover $B_{n}=\left(\iota_{n}^{-1}\left(V_{n}\right)\right)^{\circ}$ and the sets $\left(\iota_{n}^{-1} V_{n}\right)_{n}$ form a countable basis of zero neighbourhoods in $X$. By the proof of [7, Proposition 3] we may assume that $G_{n}=\iota_{n}^{\prime \prime \prime \prime} F_{n}$ and $F_{n} \in\left(B_{n}\right)_{*}^{\circ}$. We define $f_{n}:=\iota_{n}^{\prime \prime \prime} g_{n}$ and-proceeding along the lines of the proof of Proposition 3.3-we obtain 
the desired conditions. Let us also point out that, in contrast to the previous case, we do not have to assume that $X$ have a continuous norm since here we play with the injectivity of $\iota_{n}^{\prime \prime \prime}$ which is a consequence of the dense range of $\iota_{n}$.

4. Mean ergodicity and contractively power bounded operators. We start with three lemmata, which for Banach spaces are proved in [7]. Since the proofs are short, for the convenience of the reader we enclose them below.

Lemma 4.1. Let $T$ be a power bounded and mean ergodic operator on a lcHs $X$ with a mean ergodic projection $P$. Then $P^{\prime}\left(X^{\prime}\right)=F\left(T^{\prime}\right)$.

Proof. Since, by definition, $P x=\lim _{n} \frac{1}{n} \sum_{k=1}^{n} T^{k} x$ we see that $T P=P T=$ $P=P^{2}$ which gives $T^{\prime} P^{\prime}=P^{\prime}$. This yields $P^{\prime}\left(X^{\prime}\right) \subset F\left(T^{\prime}\right)$. If $x^{\prime} \in F\left(T^{\prime}\right)$ then

$$
P^{\prime} x^{\prime}(x)=x^{\prime}\left(\lim _{n} \frac{1}{n} \sum_{k=1}^{n} T^{k} x\right)=x^{\prime}(x)
$$

for every $x \in X$ which gives the other inclusion.

Definition 4.2. Let $X$ be a lcHs and $T: X \rightarrow X$ a linear and continuous operator. Suppose we are given a family $\mathcal{S}$ of subsets in $X$. We say that $T$ is contractively power bounded with respect to $\mathcal{S}$ (abbreviated $\mathcal{S}$-cpb) provided it is power bounded and $T(S) \subset S$ for at least one $S \in \mathcal{S}$.

Throughout this Section $X$ will always be a separable and 1-quasi-reflexive real Fréchet space which can be represented as the reduced projective limit of a sequence of separable and 1-quasi-reflexive real Banach spaces. If we distinguish in $X$ a countable basis $\mathcal{U}$ of zero neighbourhoods according to Proposition 3.3 together with families of functionals $\left\{f_{U}\right\}_{U \in \mathcal{U}},\left\{F_{U}\right\}_{U \in \mathcal{U}}$ then we will say that $\mathcal{U}$ is a $z$-selection. And if we associate to $X^{\prime}$ a countable family $\mathcal{B}$ of bounded sets according to Proposition 3.4 together with families of functionals $\left\{f_{B}\right\}_{B \in \mathcal{B}},\left\{F_{B}\right\}_{B \in \mathcal{B}}$ then we will say that $\mathcal{B}$ is a b-selection.

Lemma 4.3. Let $\mathcal{U}$ be a z-selection on $X$ and fix $U \in \mathcal{U}$. Suppose $Q \neq 0$ is a projection from $X^{\prime \prime}$ onto the one-dimensional subspace $\left[f_{U}\right]$ with $Q\left(U_{*}\right) \subset U_{*}$. Then $Q x^{\prime \prime}=F_{U}\left(x^{\prime \prime}\right) f_{U}$.

Proof. Clearly $Q x^{\prime \prime}=H\left(x^{\prime \prime}\right) f_{U}$ and $H \in U_{*}^{\circ}$ by assumption. Moreover

$$
H\left(f_{U}\right) f_{U}=Q f_{U}=Q^{2} f_{U}=\left(H\left(f_{U}\right)\right)^{2} f_{U} .
$$

Since $Q \neq 0$ we obtain $H\left(f_{U}\right)=1$ and by Proposition 3.3 (iii) $H=F_{U}$.

Lemma 4.4. Let $T=I+R \in L(X)$ with $R$ reflexive. Suppose we have a z-selection $\mathcal{U}$ on $X$ and $T(U) \subset U$ for some $U \in \mathcal{U}$. Then $f_{U} \in F\left(T^{\prime \prime}\right)$.

Proof. Since $R^{\prime \prime} f_{U} \in X$ we have $F_{U}\left(R^{\prime \prime} f_{U}\right)=0$. By assumption $T^{\prime \prime}\left(U_{*}\right) \subset U_{*}$. Therefore $T^{\prime \prime} f_{U}=f_{U}+R^{\prime \prime} f_{U} \in U_{*}$. Moreover $F_{U}\left(f_{U}+R^{\prime \prime} f_{U}\right)=1$ and by Proposition 3.3 (ii) $R^{\prime \prime} f_{U}=0$, i.e. $f_{U} \in F\left(T^{\prime \prime}\right)$. 
Remark 4.5. The above lemma requires explicitly that $X$ be a real space since for complex ones we could only obtain $T^{\prime \prime} f_{U}=\alpha f_{U}$ for some number $\alpha$ of modulus 1 .

The proofs of the following three results are, in the Banach space setting, originally given in [7] but for the sake of convenience we present them below with necessary modifications.

Theorem 4.6. Let $X$ be a separable and 1-quasi-reflexive real Fréchet space and take a z-selection $\mathcal{U}$ in $X$. Then any $\mathcal{U}$-cpb operator is mean ergodic.

Proof. Suppose $T: X \rightarrow X$ is a $\mathcal{U}$-cpb operator and $T(U) \subset U$ for some zero neighbourhood $U$ of the (countable) z-selection $\mathcal{U}$. By Proposition 2.3 we may assume that $T=I+R$ with $R$ reflexive. Since by [16, Theorem 4.8] $T$ or $T^{\prime}$ is mean ergodic, we may assume that $T^{\prime}$ is mean ergodic. If $F\left(T^{\prime}\right)=\{0\}$ then $X=\overline{(I-T) X}$ (use Hahn-Banach) and by [1, Theorem 2.4] $T$ is mean ergodic. Therefore we assume $F\left(T^{\prime}\right) \neq\{0\}$ and define

$$
P x^{\prime}=\lim _{n \rightarrow+\infty} \frac{1}{n} \sum_{k=1}^{n} T^{\prime k} x^{\prime}, \quad x^{\prime} \in X^{\prime} .
$$

Since $P$ is a projection onto $F\left(T^{\prime}\right)$ we see that it is non-trivial. By mean ergodicity of $T^{\prime}$ and [1, Theorem 2.4] we have a decomposition

$$
X^{\prime}=F\left(T^{\prime}\right) \oplus \overline{R^{\prime} X^{\prime}} .
$$

Moreover by Lemma $4.4 f_{U} \in F\left(T^{\prime \prime}\right)$ and so $F\left(T^{\prime \prime}\right)=F(T) \oplus\left[f_{U}\right]$. The formula

$$
Q\left(x^{\prime \prime}\right):=F_{U}\left(P^{\prime} x^{\prime \prime}\right) f_{U}, \quad x^{\prime \prime} \in X^{\prime \prime}
$$

defines a non-zero projection with $Q\left(U_{*}\right) \subset U_{*}$ and $Q\left(f_{U}\right)=f_{U}$. By Lemma 4.3 $Q x^{\prime \prime}=F_{U}\left(x^{\prime \prime}\right) f_{U}$ and $\operatorname{ker} Q=X$ which yields ker $P^{\prime} \subset X$. By Lemma 4.1 we have

$$
X^{\prime \prime}=F\left(T^{\prime \prime}\right) \oplus \operatorname{ker} P^{\prime}=\left[f_{U}\right] \oplus F(T) \oplus \operatorname{ker} P^{\prime}
$$

and therefore $X=F(T) \oplus \operatorname{ker} P^{\prime}$ (recall that $X$ is 1 -quasi-reflexive and $f_{U} \in$ $\left.X^{\prime \prime} \backslash X\right)$. Again by mean ergodicity of $T^{\prime}$ we obtain

$$
P^{\prime} x^{\prime \prime}=\lim _{n} \frac{1}{n} \sum_{k=1}^{n} T^{\prime \prime k} x^{\prime \prime}
$$

in the topology $\sigma\left(X^{\prime \prime}, X^{\prime}\right)$. If $x \in \operatorname{ker} P^{\prime}$ then

$$
\frac{1}{n} \sum_{k=1}^{n} T^{k} x \rightarrow 0
$$

in the topology $\sigma\left(X, X^{\prime}\right)$ and by [13, Theorem 1.1] (which is valid for any lcHs) also in the initial topology of $X$. Therefore $x \in \overline{(I-T) X}$. But the other inclusion is clear therefore

$$
X=F(T) \oplus \overline{(I-T) X}
$$

and by $[1$, Theorem 2.4] we obtain mean ergodicity of $T$. 
The above result together with the preceding lemmata remains valid if we consider $X^{\prime}$ with a b-selection $\mathcal{B}$ and $\mathcal{B}$-cpb operators. Therefore we can derive another consequence.

Theorem 4.7. Let $X$ be a separable and 1-quasi-reflexive real Fréchet space or its dual and take a b-selection $\mathcal{B}$ in $X^{\prime}$. Then any $\mathcal{B}$-cpb operator is mean ergodic.

Theorem 4.8. Let $X$ be a separable and 1-quasi-reflexive real Fréchet space.

(1) If $\mathcal{U}$ is a z-selection in $X$ then the adjoint of any $\mathcal{U}$-cpb operator is mean ergodic.

(2) If $\mathcal{B}$ is a b-selection in $X^{\prime}$ then the adjoint of any $\mathcal{B}$-cpb operator is mean ergodic.

Proof. Both cases are proved analogously with the only difference that in order to obtain (1) we use Theorem 4.6 and to obtain (2) we apply Theorem 4.7. Therefore we show only the first case. By Proposition 2.3 we may assume that $T=I+R$ with $R$ reflexive. Moreover let $T(U) \subset U$ for some $U \in \mathcal{U}$. By Theorem $4.6 T$ is mean ergodic so we may define

$$
E x:=\lim _{n \rightarrow+\infty} \frac{1}{n} \sum_{k=1}^{n} T^{k} x .
$$

Observe that by Lemma $4.1 E^{\prime}$ is a projection onto $F\left(T^{\prime}\right)$ and $E^{\prime \prime}$ is a projection into $F\left(T^{\prime \prime}\right)$. In particular $E^{\prime \prime} f_{U} \in F\left(T^{\prime \prime}\right)$. Now observe that by Lemma 4.4 $f_{U} \in F\left(T^{\prime \prime}\right)$ and the decomposition $X^{\prime \prime}=X \oplus\left[f_{U}\right]$ yields $F\left(T^{\prime \prime}\right)=F(T) \oplus\left[f_{U}\right]$. Hence $E^{\prime \prime} f_{U}=y+\alpha f_{U}$ with $y \in F(T)$. Consequently $E^{\prime \prime} y=y$ by the fact that $\left.E^{\prime \prime}\right|_{X}=E$. Recall that by Proposition 3.3 $F_{U} \in X^{\perp}$ therefore we get

$\alpha=F_{U}\left(\alpha f_{U}+y\right)=F_{U}\left(E^{\prime \prime} f_{U}\right)=F_{U}\left(E^{\prime \prime} E^{\prime \prime} f_{U}\right)=F_{U}\left(\alpha^{2} f_{U}+(\alpha+1) y\right)=\alpha^{2}$.

Case (i): $\alpha=1$. Since $E^{\prime \prime} f_{U} \in U_{*}$ and $F_{U}\left(E^{\prime \prime} f_{U}\right)=1$, by Proposition 3.3 (ii) we obtain $E^{\prime \prime} f_{U}=f_{U}$. By mean ergodicity of $T$ together with [1, Theorem 2.4] we obtain

$$
X^{\prime \prime}=F(T) \oplus \overline{(I-T) X} \oplus\left[f_{U}\right]=F\left(T^{\prime \prime}\right) \oplus \overline{\left(I-T^{\prime \prime}\right) X^{\prime \prime}}
$$

(recall that $\left.R^{\prime \prime} X^{\prime \prime}=R X\right)$. By [1, Theorem 2.4] together with [12, Chapter VIII, $\S 39,3(6)] T^{\prime \prime}$ is mean ergodic. Thus $E^{\prime \prime} x^{\prime \prime}=\lim _{n} \frac{1}{n} \sum_{k=1}^{n}\left(T^{\prime \prime}\right)^{k} x^{\prime \prime}$ (the limit being taken in the topology $\left.\beta\left(X^{\prime \prime}, X^{\prime}\right)\right)$. Consequently

$$
E^{\prime} x^{\prime}=\sigma\left(X^{\prime}, X^{\prime \prime}\right)-\lim _{n} \frac{1}{n} \sum_{k=1}^{n}\left(T^{\prime}\right)^{k} x^{\prime}
$$

and by [13, Theorem 1.1] we obtain the above convergence in the topology $\beta\left(X^{\prime}, X\right)$. Hence $T^{\prime}$ is mean ergodic.

Case (ii): $\alpha=0$. We have $E^{\prime \prime} f_{U} \in F(T)$ and so $E^{\prime \prime}\left(F\left(T^{\prime \prime}\right)\right)=F(T)$. Let $x^{\prime \prime} \in F\left(T^{\prime \prime}\right)$ and take $x:=E^{\prime \prime} x^{\prime \prime} \in F(T)$. Since $F\left(T^{\prime}\right)$ always separates $F(T)$ we can find $x^{\prime} \in F\left(T^{\prime}\right)$ such that $x^{\prime}(x) \neq 0$. By Lemma $4.1 E^{\prime} x^{\prime}=x^{\prime}$ therefore

$$
x^{\prime \prime}\left(x^{\prime}\right)=x^{\prime \prime}\left(E^{\prime} x^{\prime}\right)=E^{\prime \prime} x^{\prime \prime}\left(x^{\prime}\right)=x^{\prime}(x) \neq 0 .
$$


This shows that $F\left(T^{\prime}\right)$ separates $F\left(T^{\prime \prime}\right)$ and by Sine's Criterion [16, Theorem 5.1] $T^{\prime}$ is mean ergodic.

\section{Main result}

Theorem 5.1. Let $X$ be a separable and 1-quasi-reflexive real Fréchet space which can be represented as the reduced projective limit of a sequence of separable and 1-quasi-reflexive Banach spaces. There exists a countable b-selection $\mathcal{B}$ in $X^{\prime}$ so that $\mathcal{B}=\left\{V_{n}^{\circ}: n \in \mathbb{N}\right\}$ and $\mathcal{V}:=\left\{V_{n}: n \in \mathbb{N}\right\}$ is a zero neighbourhood basis in $X$. Moreover all $\mathcal{V}$-cpb operators on $X$ as well as all $\mathcal{B}$-cpb operators on $X^{\prime}$ are mean ergodic.

Proof. Choose $\mathcal{B}$ according to Proposition 3.4. Recall that $\mathcal{B}=\left\{V_{n}^{\circ}: n \in \mathbb{N}\right\}$ for some zero neighbourhood basis $\mathcal{V}$ in $X$. Let now $T$ be a $\mathcal{V}$-cpb operator on $X$. Then $T^{\prime}$ is a $\mathcal{B}$-cpb operator on $X^{\prime}$ and by Theorem $4.8(2)$ the biadjoint $T^{\prime \prime}$ is mean ergodic on $X^{\prime \prime}$. Since $\left.T^{\prime \prime}\right|_{X}=T$ we get mean ergodicity of $T$. If now $T$ is a $\mathcal{B}$-cpb operator on $X^{\prime}$ then by Theorem 4.7 it is mean ergodic.

Remark 5.2. One can easily notice that we can consider a z-selection $\mathcal{V}$ in $X$, a b-selection $\mathcal{B}$ in $X^{\prime}$ and $\mathcal{V}$-cpb operators on $X$ together with $\mathcal{B}$-cpb operators on $X^{\prime}$ and the conclusion of the main result follows by Theorems 4.6 and 4.7. The advantage of our approach lies in the fact that we do not assume that $\mathcal{V}$ be a z-selection.

We close this Section with a final observation. Suppose $X$ is a Fréchet space with a basis. By [1, Theorem 1.4] it is reflexive if all power bounded operators on $X$ are mean ergodic. But if we additionally assume that these operators are cpb (which does not seem to be much more restrictive) then the space need not be reflexive. This shows that reflexive and non-reflexive Fréchet spaces are, in a sense, close to each other.

Open Access. This article is distributed under the terms of the Creative Commons Attribution Noncommercial License which permits any noncommercial use, distribution, and reproduction in any medium, provided the original author(s) and source are credited.

\section{References}

[1] A. A. Albanese, J. Bonet, and W. J. Ricker, Mean ergodic operators in Fréchet spaces, Ann. Acad. Sci. Fenn. Math. 34 (2009), 401-436.

[2] K. D. Bierstedt, An introduction to locally convex inductive limits, Functional Analysis and Its Applications (Nice, 1986. H. Hogbe-Nlend, ed.), pp. 35-133, ICPAM Lecture Notes, World Sci. Publishing, Singapore, 1988.

[3] P. Civin And B. Yood, Quasi-reflexive spaces, Proc. Amer. Math. Soc. 8 (1957), 906-911.

[4] Y. Cuttle, On quasi-reflexive Banach spaces, Proc. Amer. Math. Soc. 12 (1961), 936-940.

[5] N. Dunford And J. T. Schwartz, Linear Operators. Part I: General Theory, 2nd ed., Wiley-Interscience, New York, 1964. 
[6] V. P. Fonf, M. Lin, And P. Wojtaszczyk, Ergodic characterizations of reflexivity of Banach spaces, J. Funct. Anal. 187 (2001), 146-162.

[7] V. P. Fonf, M. Lin, And P. Wojtaszczyk, A non-reflexive Banach space with all contractions mean ergodic, preprint.

[8] B. V. Godun, Equivalent norms on quasireflexive spaces with a basis (Russian), Dokl. Akad. Nauk Ukrain. SSR Ser. A 1977, 778-781, 863.

[9] R. C. James, Bases and reflexivity of Banach spaces, Ann. of Math. 52 (1950), $518-527$.

[10] R. C. James, Banach spaces quasi-reflexive of order one, Studia Math. 60 (1977), $157-177$.

[11] G. Kӧтне, Topological Vector Spaces I, Springer-Verlag New York Inc., New York, 1969.

[12] G. Кӧтне, Topological Vector Spaces II, Springer-Verlag, New York-Berlin, 1979 .

[13] U. Krengel, Ergodic theorems. With a supplement by Antoine Brunel, de Gruyter Studies in Mathematics 6, Walter de Gruyter Co., Berlin, 1985.

[14] V. K. Maslyuchenko and A. N. Plichko, Quasireflexive locally convex spaces without Banach subspaces (Russian), Teor. Funktsii Funktsional. Anal. i Prilozhen. 44 (1985), 78-84; translation in J. Soviet Math. 48 (1990), 307-312.

[15] R. Meise And D. Vogt, Introduction to Functional Analysis, Clarendon Press, Oxford, 1997.

[16] K. PiszczeK, Quasi-reflexive Fréchet spaces and mean ergodicity, J. Math. Anal. Appl. 361 (2010), 224-233.

[17] I. Singer, On bases in quasi-reflexive Banach spaces, Rev. Math. Pures Appl. (Bucarest) 8 (1963), 309-311.

[18] I. Singer, Bases and quasi-reflexivity of Banach spaces, Math. Ann. 153 (1964), 199-209.

[19] L. Sucheston, Problems. Probability in Banach Spaces, Lecture Notes in Math. 526, Springer-Verlag, Berlin, 1976.

[20] M. Valdivia, Bases and quasi-reflexivity in Fréchet spaces, Math. Nachr. 278 (2005), 712-729.

\section{KrzyszTof PiszczeK}

Faculty of Mathematics and Computer Science,

Adam Mickiewicz University,

Poznań ul. Umultowska 87, 61-614 Poznań, Poland

e-mail: kpk@amu.edu.pl

Received: 26 January 2010

Revised: 20 September 2010 